\title{
Téoros
}

Revue de recherche en tourisme

\section{Une typologie des agriculteurs qui diversifient leurs activités vers le tourisme Une approche phénoménologique}

\section{Laurent Bourdeau, Maurice Doyon et Dorothée Donne}

Volume 20, numéro 1, printemps 2001

URI : https://id.erudit.org/iderudit/1071913ar

DOI : https://doi.org/10.7202/1071913ar

Aller au sommaire du numéro

Éditeur(s)

Université du Québec à Montréal

ISSN

0712-8657 (imprimé)

1923-2705 (numérique)

Découvrir la revue

Citer cet article

Bourdeau, L., Doyon, M. \& Donne, D. (2001). Une typologie des agriculteurs qui diversifient leurs activités vers le tourisme : une approche phénoménologique. Téoros, 20(1), 63-71. https://doi.org/10.7202/1071913ar d'utilisation que vous pouvez consulter en ligne. 


\title{
Une typologie des agriculteurs qui diversifient leurs activités vers le tourisme
}

\author{
Une approche phénoménologique
}

\section{Laurent Bourdeau, Maurice Doyon et Dorothée Donne}

L'agriculture en Occident, et particulièrement en Amérique du Nord, a épousé un modele productiviste qui mène à la concentration des entreprises agricoles. Bien que ce modele ait permis de produire une quantité d'aliments toujours croissante à des coûts réels qui vont en diminuant, certaines contraintes font en sorte qu' un nombre de plus en plus important de producteurs agricoles cherchent des solutions pour accroitre les revenus de ce modele (Doyon er al, 2001). L'intégration d'activités complémentaires aux activites agricoles qui on une forte valeur ajoutée semble être l'alternative choisie par un nombre de plus en plus important de producteurs agricoles. Dans un tel contexte, le tourisme apparait comme une alternative ayant un très fort potentiel (Dion, 2001).
$\mathbf{A}$ fin de mieux comprendre les raisons qui conduisent certains agriculteurs à se tourner vers le tourisme pour diversifier leur's activités, il apparaît pertinent de décrire les causes du phénomène de concentration des entreprises agricoles et de discuter des contraintes qui poussent certains producteurs agricoles â vouloir briser le cycle de la concentration, en choisissant plutôt l'intégration d'activités complémentaires.

Dans le contexte économique actuel, la réduction des coûts de production devient un important facteur de perennite des entreprises agricoles. En effet, une entreprise qui ne suit pas le mouvement se trouverait, â moyen terme, à produire à un colît supérieur au prix du marché ${ }^{1}$. Pour illustrer ce phénomène, on peut observer l'evolution du prix du porc en dollar constant (voir figure 1). On constate alors que le prix réel du porc (dollar 1998)² a chuté de façon dramatique de 1979 a 1998 . En fait, il s'agit d'une baisse de $61 \%$ du prix rél. Cette baisse de prix ne s"est pas faite au détriment de la production porcine québécoise, puisque, pendant la même période, le nombre de porcs abattus augmentait de plus de $70 \%$ au Québec. Les gains d'efficacité, qui proviennent principalement de l'innovation technologique, ont donc été très importants dans le secteur porcin.

Le modèle productiviste conduit donc à une baisse des marges unitaires, ce qui incite l'agriculteur à produire un plus gros volume lui permettant de bénéficier $\mathrm{d}^{+} 6 \mathrm{co}-$ nomies d'echelle et d'obtenir un revenu satisfaisant. Il s'agit done d'une fuite en avant où l'agriculteur produit toujours davantage, à plus bas prix, tout en compensant partiellement la baisse réelle des prix par des gains d'efficacité et en maintenant son revenu par l'augmentation des

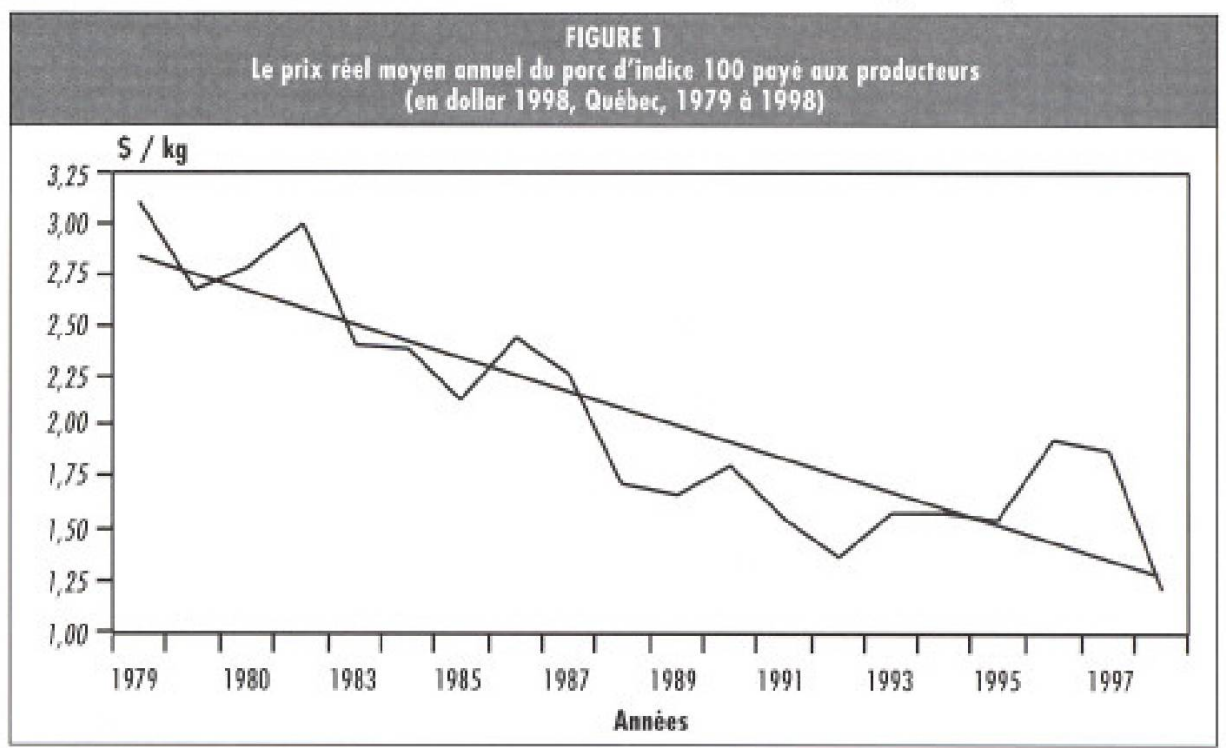

Source ; Anowole stotisnique porcin, GREPA, 1959. 
volumes produits. Toutefois, l'expansion des entreprises agricoles fait de plus en plus face à des contraintes environnementales et de production ${ }^{3}$. Devant ces contraintes, plusieurs exploitations se trouvent à moyen terme confrontées à trois choix : quitter le secteur, acquerir une autre exploitation ou encore développer des activités complémentaires à̀ valeur ajoutée.

Certains agriculteurs n'agissent pas nécessairement en réaction à des contraintes, mais refusent la fuite en avant proposée par le modèle productiviste. Pour ces derniers, la production industrielle de biens de plus en plus uniformes, en grande quantité et à faible prix, ne correspond plus à leur conception de l'agriculture. Ces agriculteurs visent alors à modifier leurs activités agricoles. L'agriculture biologique et les produits du terroir s"inserivent d'ailleurs dans cette démarche. Cette offre modifiée peut alors prendre la forme de transformation des produits de la ferme, d'utilisation de nouvelles techniques de production ou encore la valorisation de l'environnement et des produits de la ferme par le biais de l'agrotourisme.

\section{La commercialisation des activités agricoles et touristiques}

Dans le système agricole actuel, on distingue deux types d'entreprises agricoles (Fougerousse, 1996) : celles qui remettent en question le phénomène de concentration des entreprises agricoles en modifiant leurs activités agricoles et celles qui transforment leurs entreprises en participant au phénomène de concentration.

La diversification des activités par l'agrotourisme confere aux entreprises qui remettent en question le phénomène de concentration des entreprises agricoles un rôle de producteur d'activités touristiques. C'est en effet à partir du système agricole que l'offre touristique est proposée aux touristes ou aux résidants ${ }^{4}$. Cette offre touristique devrait d'ailleurs être créée comme une offre complémentaire à l'activité agricole (Union des producteur's agricoles, 1999) permettant aux touristes de se familiariser au monde agricole.
Bien que l'offre d'activités touristiques en milieu agricole doive être représentative du monde agricole, on peut se demander quelles sont les motivations des agriculteur's à se tourner vers ce type de diversification. Si la première motivation apparâit avant tout économique, l'offre d'activités touristiques par une exploitation agricole apparất également un moyen, pour le responsable agricole, de transmettre un message agricole (Disez, 1997).

Considérant que chaque agriculteur cherche à créer des liens avec son environnement externe (Lussato, 1972), les entreprises agricoles qui commercialisent des activités touristiques devraient chercher à créer des liens avec l'industrie touristique. En conséquence, afin de commercialiser leurs activités touristiques, les entreprises agricoles ne devraient pas se limiter à leur système agricole, mais interagir avec les intervenants touristiques. Nous ne connaissons aucune recherche scientifique qui nous permette de comprendre cette interaction.

Cette recherche a trois objectifs qui permettront de circonscrire cette interaction. Le premier objectif consiste à comprendre les motivations qui conduisent certains agriculteurs à se tourner vers le tourisme pour diversifier leurs activités. Le deuxième vise à comprendre les interactions entre le monde agricole et le monde touristique quand des entreprises agricoles commercialisent des activités touristiques. Finalement, l'étude des motivations des agriculteurs qui se tournent vers l'offre touristique vise à dresser un profil des agriculteurs qui ont choisi la diversification de leurs activités.

\section{Méthodologie}

Les données de cette recherche ont été recueillies auprès d'un échantillon de vingt-huit agriculteurs. Compte tenu du caractère exploratoire de la recherche, une technique qualitative a té utilisée pour la collecte des données. Une approche phénoménologique a été choisie pour analyser le phénomène étudié. De façon plus précise, les données ont ếté recueillies à l'aide d'entrevues en profondeur en utilisant des questions ouvertes ${ }^{5}$. Cette méthode nous apparaît appropriée puisque nous cherchions à découvrir les raisons (Churchill, 1991) ou les motivations (Perrien et al., 1984) qui conduisent les agriculteurs à se tourner vers l'agrotourisme. De plus, des contraintes physiques nous étaient imposées par la période de collecte des données. En effet, l'enquête étant réalisée pendant le mois d'août, il aurait été difficile, voire impossible, de demander aux agriculteurs de se déplacer (par exemple pour participer à un groupe de discussion). Rappelons que, pendant cette période, les agriculteurs sont occupés soit par les travaux agricoles, soit par les touristes. Les entrevues réalisées dans le cadre de l'étude ont duré en moyenne une heure trente chacune.

Les entrevues \& face à face » comportent de nombreux avantages. Notamment, elles permettent au chercheur de vérifier l'exactitude de certaines réponses et d'obtenir des renseignements qui ne pourraient être divulgués par l'utilisation d'une recherche quantitative (Gauthy et al., 1998). Par contre, une des principales faiblesses de ce type d'enquête réside dans la validité et la fidélité des entrevues (Perrien et al., 1984), Comme la présente recherche exploratoire ne vise pas à réaliser une étude probabiliste des motivations poussant les agriculteurs à se tourner vers l'agrotourisme, la validité et la fidélité des instruments de mesure utilisés ne peuvent donc pas affecter sa démarche scientifique.

L'échantillon de cette recherche est composé d'agriculteurs dont les exploitations agricoles sont représentatives de la région et ce, tant au plan géographique et agricole que touristique. Les agriculteurs interrogés ont leur site d'exploitation dans la région touristique de Chaudière-Appalaches et cherchent, par leurs activités, à attirer les touristes québécois ou étrangers sur leur ferme. Plus spécifiquement, les fermes échantillonnées sont situées dans les Municipalités régionales de comtés (MRC) de Lotbinière, de Bellechasse, de la Rive-sud et de Montmagny-L'Islet ${ }^{6}$. Les activités touristiques prennent différentes formes : le gîte touristique, la restauration mettant en valeur les produits de la ferme ou la visite de l'exploitation agricole. 
Précisons que dans le choix des entreprises étudiées, nous n'avons pas retenu les exploitations qui n'offrent pas d'activités aux touristes. Bien que notre échantillon ne soit pas statistiquement représentatif de la population visée et ne représente pas nécessairement l'ensemble des caractéristiques de la population (Abdelahim, 1998), il rassemble néanmoins l'ensemble des produits formant l'offre touristique de la région touristique de Chaudière-Appalaches. Notons qu'en plus de s'intéresser au tourisme, certaines exploitations agricoles étudiées cherchent également à attirer la clientèle qui habite dans la région immédiate de leur exploitation. On peut d'ailleurs observer qu'environ la moitié des fermes propose la vente de produits et des visites à la ferme. Pour ces agriculteurs, l'agrotourisme est une activité récréotouristique.

Les entrevues se sont déroulées à partir de trois indicateurs: l'exploitation agricole et l'activité touristique offerte sur l'exploitation agricole; les relations entre les activités agricoles et touristiques; la gestion des activités touristiques par l'exploitation agricole. Finalement, notons que les responsables de l'activité touristique interrogés sur l'exploitation agricole possèdent, pour la majorité, une certaine expérience personnelle. Parmi les exploitants rencontrés, quatre ont un diplome en agriculture, six ont suivi des sessions en agriculture et vingt et un n'ont aucune formation agricole. Parmi ces derniers, treize sont issus du milieu agricole. Parallèlement, dix d'entre eux ont suivi une formation en tourisme.

\section{Analyse des résultats}

Cette recherche identifie quatre catégories d'entreprises agricoles offrant des activités touristiques. Il n'existe aucun lien entre l'âge de la ferme et son appartenance à l'une de ces catégories. De plus, pour chacune des catégories, on constate que la majorité des activités touristiques est effectuée sur une base saisonnière. Ces activités touristiques se produisent généralement pendant le déroulement des activités agricoles.

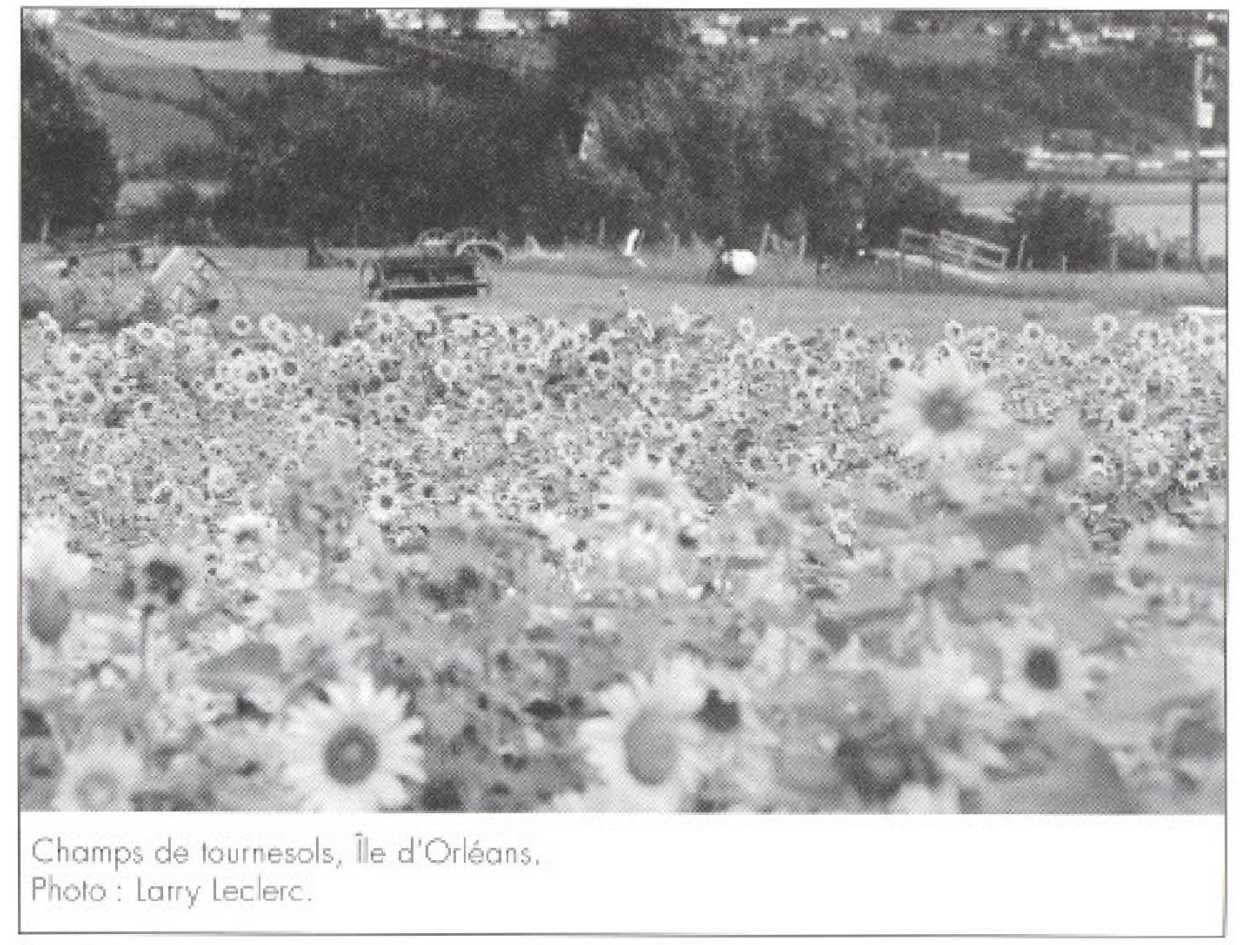

\section{Les professionnels du tourisme en milieu rural}

L'exploitation agricole ef l'activité touristique. Pour les entreprises de cette catégorie, les activités touristiques sont intégrées aux activités de la production agricole et sont tributaires d'une infrastructure aménagée sur le site de l'exploitation. On peut qualifier ces agriculteurs de professionnels parce que les activités touristiques sont intégrées à un système commercial organisé qui fait partie des activités agricoles. Parmi ces activités touristiques, on trouve les visites à la ferme, l'offre de gîte, la restauration et les camps de vacances; les producteurs de petits fruits, de pommes, d'animaux exotiques, de vaches laitières, de moutons, de pores et de produits de l'érable.

Tous les producteurs agricoles de cette catégorie ont un revenu agricole jugé suffisant pour maintenir l'entreprise agricole en production. L'intégration des activités touristiques est donc une valeur ajoutée qui permet d'augmenter les revenus des exploitants. On remarque que ces producteurs sont tous membres d'une association liée à leur production. Les activités touristiques sont gérées par le responsable de la ferme, un membre de la famille ou des salariés saisonniers. Les motivations de ces agriculteurs face aux activités touristiques, outre leur valeur économique, s'appuient sur la volonté de communiquer leur savoir et leur métier, mais aussi de faire vivre aux touristes une expérience agricole.

L'agriculteur veut : \& [...] être en relation avec le public, expliquer son activité, eduquer . Selon les agriculteurs, les touristes chercheraient à vivre la vie des exploitants : \& Ceux qui viement, c'est par choix d'aller sur whe ferme. Ils le savent, il y a du bruit, des odeurs [...] c'est la fermes.

De façon générale, dans les fermes de la catégorie professionnels du tourisme en milieu rural, le message transmis aux touristes par le biais des activités touristiques concerne la réalité de l'agriculture. On cherche alors à transmettre aux touristes le rồle joué par les agriculteurs à trä vers le système de production des aliments.

Proteger la terre, faire connaître l'agriculture [...] donner aux touristes les bonnes données $[\ldots]$ de l'agriculture et de l'elevage. Montrer les atouts et les contraintes rélles du vécu de l'agriculteur: Les gens pensent que l'agricalture, c'est tran- 
quille, c'est la campagne, relaxe or, c'est pas ça [...] Leur faire goûter quelque chose de different, leur faire vivre une expérience [...] Montrer le lien entre l'agriculture et l'assiette et que la vie à la campagne inclut le travail de l'agriculteur qui est important.

Les clientèles touristiques ciblées par les entreprises de la catégorie professionnels peuvent varier selon le type d'activités touristiques offertes. Par exemple, les gastronomes sont ciblés quand des produits de la ferme sont offerts. Tous ces agriculteurs visent des groupes de touristes, mais, dans plusieurs cas, il faut réserver à l'avance puisque l'agriculteur doit consacrer l'essentiel de son temps à ses activités agricoles. Il y a donc une limite de l'offre touristique de ce groupe. Quand les infrastructures le permettent, plusieurs entreprises ciblent également les groupes d'enfants et les familles avec ou sans enfants. Les visites à la ferme sont généralement payantes, sauf pour les familles. On rentabilise alors le temps consacré aux familles par la vente de produits agricoles.

Les relations entre les activités agricoles et touristiques. Les agriculteurs de cette catégorie font la promotion de leurs actiwités touristiques en utilisant plusieurs médias aux plans local et régional. Tous ces agriculteurs sont membres de l'Association touristique régionale (ATR) et ont utilisé les médias (radiophoniques, télévisés et écrits) pour faire connaître leurs activités touristiques. De plus, tous entretiennent des relations avec des organismes ou des entreprises liés soit à l'industrie agricole (ex.: Union des producteurs agricoles [UPA]), soit à l'industrie touristique (ex. : Guide Debeur, 1'Association des camps du Québec). On constate également que la moitié des membres de cette catégorie contacte régulièrement les agences de voyages. Des relations existent également avec des agriculteurs voisins qui offrent des activités touristiques, notamment, par l'offre d'un forfait touristique, d'activités touristiques communes ou dans l'approvisionnement de marchandises destinées à la réalisation des activités touristiques.
J'achète mes produits pour la restauration chez des voisins. J'en prends partout, ils sont tous mes fournisseurs. Je ne favorise personne. C'est important, ca permet de maintenir un contaca dans cette région peu peuplée.

De façon générale, ces relations demeurent aux plans local et régional, notamment au niveau de la MRC d'appartenance.

La gestion des activités touristiques par l'exploitation agricole. Les agriculteurs de cette catégorie maîtrisent l'offre associée aux activités touristiques. D'ailleurs, pour ceux-ci, l'activité touristique dégage un revenu qui permet son autofinancement en plus d'accroître les profits de l'entreprise agricole. L'exploitant agricole a suivi une formation lui permettant de gérer les activités touristiques offertes ou a acquis une certaine expérience en tourisme. Ces agriculteurs apparaissent être en phase d'entretien de leurs activités touristiques et cherchent à se former pour mieux cibler la clientèle. En effet, plusieurs agriculteurs ont de la difficulté à communiquer en anglais ou ont de la difficulté à entrer en relation avec les gens. Dans la perspective où l'agriculteur joue un rôle direct dans l'offre touristique, la formation de ces individus pourrait améliorer la qualité de l'offre touristique.

\section{Les novices du tourisme en milieu rural}

L'exploitation agricole et l'activité touristique. Pour les entreprises de cette catégorie, les activités touristiques varient selon le type de produits agricoles. Parmi les novices, l'activite touristique prend trois formes: la visite de la ferme et la vente de produits agricoles, l'offre d'un forfait comprenant la restauration, le gite et une visite à la ferme ou l'offre d'une restauration liée à des produits du terroir. On trouve dans cette catégorie des producteurs de petits fruits, de pommes, d'animaux exotiques, de moutons, de produits de l'érable et de produits horticoles. On peut qualifier ces agriculteurs de novices parce que même s'ils intègrent les activités touristiques aux activités agricoles de leur ferme, ils ne parviennent pas à développer un système commercial organisé et rentable de leurs activités touristiques. On constate $\mathrm{d}^{+}$ailleurs que l'aménagement de leurs activités touristiques ne s'autofinance pas et ne permet pas d'assurer l'entretien de leur système agrotouristique.

La principale motivation de ces agriculteurs pour se tourner vers le tourisme s'appuie sur des considérations economiques. On cherche alors à diversifier les revenus de la ferme. Ces agriculteurs affirment que l'ajout d'activités touristiques leur permet de vendre des produits de la ferme, de créer des emplois ou d'avoir un revenu supplémentaire. La thématique du message offert aux touristes est alors principalement liée aux motivations écononiques de cette catégorie d'entreprises. En effet, on note que les producteurs ont pour objectif de faire connaitre la production afin de: * [...] vendre leurs produits $[. .$.$] et de par-$ tager l'amour des bêtes et de la nature $\%$.

D'ailleurs, quand on interroge un des agriculteurs sur le message transmis aux touristes à travers l'expérience vécue sur la ferme, on peut clairement identifier la présence de considérations économiques.

\section{Je veux qu'ils vivent l'agriculture, montre que l'auf vient de la poule et que, s'il est fécondé, ça devient un poussin ; que l'agriculture c'est du travail er que pour avoir quelque chose de bien, ilfaut payer plus cher. I'achète du beurre et non de la margarine.}

Aucune clientèle touristique n'est clairement ciblée par les entreprises de cette catégoric. Toutes les catégories de touristes sont attendues, sans préférence. Les visites a la ferme sont payantes et, pour les groupes, se font sur réservation. Comme pout les entreprises agricoles de la catégorie professionnels, les visites à la ferme sont gratuites pour les familles, alors que le temps consacré à celles-ci est rentabilisé par la vente de produits agricoles.

Les relations entre les activités agricoles et touristiques. Les producteurs de cette catégorie cherchent avant tout à se différencier en offrant une activité spécialisée (ex. : équitation). La moitié d'entre eux fait 
la promotion de ses activités touristiques en utilisant les médias radiophoniques locaux et la télévision communautaire. L'autre moitié compte uniquement sur le bouche-à-oreille. Pour faire connaître leurs activités touristiques, tous les fermiers de la catégorie novices ont écrit des articles dans les journaux locaux et ont distribué un dépliant décrivant ces activités au bureau d'information touristique de l'ATR. En outre, ces agriculteurs sont, pour la plupart, membres de la Fédération des Agricotours, ils sont présents dans le Guide du Routard et ils entretiennent des relations avec les agences de voyages. Les relations avec les agriculteurs voisins sont peu nombreuses. Deux agriculteurs de cette catégorie rapportent avoir fait des tentatives de forfaits avec des agriculteurs voisins qui ont été vouées à l'échec par: [...] manque de motivation de l'Office du tourisme. Je devais tout organiser [...] Trop de lemps passé à organiser ces activites.

L'organisation de la production agricole de ces entreprises exige que les agriculteurs y consacrent la majorité de leur temps. Cette situation ne laisse que peu de temps à consacrer à la gestion et à la commercialisation des activités touristiques: * J'ai un boulot à coté qui me prend 45 heures par semaine [...] Je n'ai pas de formation en tourisme et je ne m'y investis pas à temps plein $\$$

De façon générale, les relations demeurent au niveau local. Seule la relation avec l'ATR permet de communiquer avec des touristes à l'extérieur de la région.

La gestion des activités touristiques par l'exploitation agricole. Les activités touristiques étant basées uniquement sur la production agricole et la qualite de l'accueil, ces agriculteurs gèrent la commercialisation de leurs activités touristiques uniquement à partir d'une vision agricole. Dans ce type de gestion, les activités touristiques ne sont que secondaires, Les novices n'ont pas ou peu de formation en tourisme.

C'est jamais manvais d'être formé, mais i'y suis arrive sans formation [...] On a des formations en tourisme et des formations en agriculture, mais pas les deux ensemble. Or, on a besoin d'aide, besoin d'appuifinancier. Il faut s'y metre ensemble.

Tel que mentionné précédemment, ces agriculteurs semblent avoir des contraintes dans l'organisation du temps de travail. Par conséquent, ils aimetaient savoir comment organiser l'offre touristique, mais ne possèdent pas les ressources financières pour organiser adéquatement la commercialisation de leurs activités touristiques.

l... Jee serait indispensable, mais je $n$ 'ai pas les moyens, et puis, l'employé affecté au tourisme demandera sa paie en fin de semaine l.../ On ne peut pas le payer [...] Rien ne peut nous remplacer. Faut tre capable de detecter les besoins des touristes et de répondre instinctivement à leur curiosité.

La méconnaissance de leur clientèle et l'absence d'une stratégie de commercialisation de leur's activités touristiques a des conséquences sur le développement de partenariats avec d'autres intervenants de l'industrie touristique.

On est très mal informe $[\ldots$. on ne sait pas ce qui se fait, qui sont les imtervenants, qui somi les responsables [...] On aimerait avoir des contacts par l'intermédiaire d'une personne responsable de l'agrotourisme dans le secteur [...] On n'est pas connu par les agriculteurs [...] Il manque de personnes neutres pour etre responsable du développement agrotouristique dans la région.

Dans le cas des agriculteurs, les normes legislatives associées au type de production agricole et le cout de la signalisation touristique sur les routes qui serait nécessaire pour faire la promotion de leur ferme sont des facteur's qui limitent le développement de leurs activités touristiques.

\section{Les initiateurs du tourisme en milieu rural}

L'exploitation agricole ef l'activité touristique. Pour les entreprises de cette catégorie, les activités touristiques sont, au départ, associées à des activités récréotouristiques qui s'appuient sur l' " autocueillette $s$ de produits agricoles ou la visite des lieux de production des produits agricoles. En fäit, l'activité est articulée autour des produits agricoles destinées en priorité aux résidants. On trouve dans cette catégorie des producteurs de pommes, de fruits et de légumes biologiques, de miel, de canards et de produits de l'Érable. Les coûts d'opération des activités récréotouristiques offertes sont peu élevés. Souvent, ils se limitent à la simple installation d'un poste de vente ou d'accueil. On peut qualifier ces agriculteurs d'initiateurs parce que leurs activités avaient au départ un caractère récréotouristique : Ils cherchaient dans un premier temps à offrir la possibilité aux gens de la région de se procurer des produits agricoles tout en vivant une activité de loisirs. Puis, dans un deuxième temps, ces producteurs agricoles désirent élargir l'offre de leurs activités aux touristes.

La principale motivation de ces agriculteurs pour se tourner vers les activités touristiques réside dans la diversification des clientèles qui peuvent acheter leurs produits agricoles. D'un point de vue économique, ces agriculteurs ne cherchent pas directement à rentabiliser l'activité offerte, mais les produits agricoles produits sur la ferme. En plus de vendre ses produits, l'agriculteur cherche aussi à accroître ses revenus. La thématique du message de l'initiateur cherche à faire vivre l'agriculture, mais à partit du produit agricole. L'expérience et l'animation n'apparaissent pas centrales dans l'offre touristique.

[...] Eduquer sur la production agricole effectué ici et sur les produis que l'on pewt en retirer: Que les touristes repartent en se disant qu'ils ont fait we visite agréable. qu'ils aient le goût de revenir et d'en parlet [...] On est une ferme réellement organique. Il faut savoir que l'on peut continuer à culiver des petits fruits comme avant avec des bons résultats. Cela permet d'avoir une meilleure alimentation et un meillear mode de vie [...] Il faut que les visiteurs sachent que ça vaut la peine 
d'entrer dans le pays, de delaisser la route principale.

Pour les agriculteurs de cette catégorie, il est important aussi de faire connaître la qualité de la production et de transmettre des connaissances associés aux produits agricoles.

\section{[...] Faire connaître la fraîcheur et la qualité du produit [...] Augmenter les connaissances des visiteurs et répondre à leur curiosité [... ] leur apprendre la manipulation du fruit ; c'est fragile, c'est vivant, il faut en prendre soin.}

Si la clientèle visée était avant tout composée de résidants de la région, mais elle a tendance à s'clargir. Au fur et à mesure que se développe un intérêt pour l'industrie touristique, les initiateurs s'intéressent aux touristes provenant de l'extérieur de la région et aux touristes étrangers.

Nous ciblons la clientèle française du Périgord qui va chez le producteur et achète ses produits, ils font de même au Québec. On cherche les amants de la gastronomie et de la bonne chair et qui ont les moyens financiers [... J les personnes sensibilisées aux soins thérapeutiques de nos productions.

On constate que même si l'offre d'activités touristiques en est à ses débuts pour ces agriculteurs, ils cherchent néanmoins à segmenter la clientèle visée. En fait, en partant du produit agricole, ils cherchent à conserver les résidants tout en ajoutant des touristes intéressés par une visite à la ferme dans le but de se procurer des produits agricoles. Chez ces entreprises, les visites à la ferme sont gratuites.

Les relations entre les activités agricoles et touristiques. Afin de promouvoir leurs activités touristiques, les agriculteurs de cette catégorie misent sur les médias écrits, notamment la publication d'articles dans les journaux locaux et régionaux, ainsi que sur une présence à des salons. Tous ces agriculteurs affirment que le meilleur outil reste le bouche-à-oreille. La radio et la té- lévision sont très peu utilisees. Les agriculteurs de cette catégorie possèdent peu de matériel promotionnel comme des dépliants expliquant leurs activités. Ces agriculteurs sont membres de l'UPA, mais ne sont pas membre de l'ATR de leur région. Ils n'ont donc pas encore intégré le réseau des intervenants touristiques.

La gestion des activités touristiques par l'exploitation agricole. La gestion des activités touristiques des initiateurs n'a que peu de liens avec les intervenants touristiques. Soulignons que la décision d'offrir des activités touristiques est nouvelle pour la majorité de ces producteurs agricoles. Ces agriculteurs ont besoin d'information concernant l'industrie touristique et de rencontres leur permettant de mieux connaître les expériences vécues par les autres entreprises agricoles qui offrent des activités touristiques. «On a beaucoup à apprendre des vergers et des érablières pour pouvoir échanger $[\ldots]$ même si la première experience en tourisme êtait manvaise, je suis prête à me réinvestir car c'est mon idee $\%$.

Les initiateurs souhaitent recevoir une formation pour accroitre leurs chances de réussite. Ce besoin de formation ne serait pas limité au tourisme: a dans tous les domaines, il ne faut janais tomber dans l'habitude, toujours innover $\%$.

Comme dans les deux catégories précédentes, les initiateurs ont des contraintes d'organisation du temps de travail, axé presque uniquement autour des activités agricoles. En plus de cette contrainte de temps, les initiateurs ne savent pas comment faire la promotion de leurs activités touristiques et n'ont pas d'argent à investir dans la signalisation touristique. Pour eux, les activités touristiques ne sont donc pas encore totalement intégrées aux activités agricoles et génèrent peu de revenus supplémentaires. Toutefois, il existe une volonté de la part de ces agriculteurs d'accroître l'importance des activités touristiques dans leur entreprise.

\section{Les suiveurs du tourisme en milieu rural}

L'exploitation agricole et l'activité touristique. Pour les agriculteurs de cette catégorie, les activités touristiques représentent une façon d"autofinancer l'entreprise agricole. Il s'agit, pour la plupart, de très petites exploitations agricoles qui nécessitent de la part des propriétaires l'injection de capitaux extérieurs, comme le travail hors ferme. La diversification des activités de l'entreprise permet donc à cette dernière de poursuivre les activités agricoles en évitant d'accroitre la production et de modifier le statut d'agriculteur à temps partiel des exploitants. Le produit touristique propose est très homogène et uniquement fondé sur la visite à la ferme. Les aménagements destinés aux visites touristiques ne sont pas termines ou inexistants. Ces fermiers en sont à leurs débuts dans la diversification et les activités touristiques. Ils sont également en réflexion quant à leurs chances de réussite ou de poursuite des activités touristiques.

La principale motivation des suiveurs se fonde donc sur des considérations économiques visant le maintien dans le temps de l'entreprise agricole. Une seule de ces entreprises affirme rechercher le contact avec le public par le biais de ses activités touristiques. De plus, la moitié des entreprises de cette catégorie ne fait aucune dépense de promotion et les dépenses de l'autre moitié des entreprises sont inférieures a $200 \$$. On peut donc constater que l'effort de promotion des activités touristiques est pratiquement nul.

Pour ces entreprises, les visites à la ferme sont payantes et le nombre de touristes est, en général, faible : de 10 à 100 visiteurs par an. La thématique du message cherche à faire connaître le produit agricole : « Faire connaitre l'animal [...] Montrer comment on maintient un animal à l'état sauvage, même dans un élevage. Les encourager à manger la viande de bison $[\ldots]$ s.

Les relations entre les activités agricoles el louristiques. Les agriculteurs de cette catégorie visent tous les touristes et les résidants de la région, sans distinction. Comme nous l'avons mentionné précédemment, au plan touristique, il y a peu de démarches. Un seul agriculteur a déjà accordé une entrevue à la radio, deux à des 
journaux locaux et deux entreprises font parties de l'ATR. Les entreprises de cette catégorie ne sont pas membres d'associations agricoles.

La gestion des activités touristiques par l'exploitation agricole. Les agriculteurs de cette catégorie ne maîtrisent pas la gestion ni la commercialisation de l'offre associé aux activites touristiques. Cette absence de mâtrise peut être liée à une méconnaissance de l'industrie touristique ou à un manque d'habileté en gestion. Les entreprises de la catégorie suiveurs seraient toutes en phase de réflexion quant à leur avenir dans l'industrie touristique. Les entrepreneurs se sentent seuls dans la commercialisation de leurs activités touristiques.

Je reste très passif, je ne fais ancane démarche particulière [...] On doit faire face à la menace de l'environnement immediat avec l'agriculture intensive et ses impacts visuels, de pollution, auditifset d odeurs $[. . . / \mathrm{La}$ région est laissée pour comple au niveau de l'aide touristique par rapport aux autres régions du Québec.

\section{Discussion}

Une des principales contributions de cette recherche se situe dans la proposition d'une typologie des agriculteurs qui diversifient leurs activités agricoles en se tournant vers le tourisme. Les quatre catégories d"entreprises se différencient selon le degré d'intégration des activités touristiques de l'agriculteur au système de commercialisation de l'industrie touristique (voir figure 2). Les professionnels, qui intègrent les activités touristiques ct agricoles ; les novices, en phase de développement de leurs activités touristiques ; les initiateurs, qui sont au début de la phase d'intégration ; et les suiveurs, en questionnement sur la mise en place d'une telle activité, On constate qu'une des motivations communes a diversifier ses activités agricoles vers des activités touristiques réside dans des considérations économiques.

La catégorie professionnelle du tourisme regroupe des activités touristiques qui visent à représenter la réalité agricole aux touristes.
Une des grandes forces du systeme d'exploitation des professionnels réside dans l'organisation commerciale des activités touristiques en lien avec les activités agricoles. L'expérience touristique se crée alors à partir d'une réalité non transformée, celle de la vie sur la ferme. Ces activités touristiques visent tant les familles que les groupes, mais une contrainte associée au temps semble exister lorsque les visiteurs sont nombreux. Les agriculteurs de cette catégorie parviennent à intégrer les activités touristiques et agricoles à l'intérieur de leur système de gestion, mais aussi à s'intếgrer au système de commercialisation des produits agricoles et touristiques. Ces entreprises sont donc celles qui sont les plus développées en termes de commercialisation d'activités agrotouristiques et de tourisme en milieu rural.

La catégorie novices du tourisme en agriculture regroupe des activités touristiques périphériques aux activités agricoles. Ce système d'exploitation des activités touristiques est plus ou moins organisé, non seulement au plan du contenu de l'offre touristique, mais également à celui de sa commercialisation. Soulignons qu'au plan de la commercialisation, aucune clientèle n'est véritablement ciblée. Les agriculteurs de cette catégorie se sont tournés vers l'offre touristique pour diversifier leurs sources de revenus sans toutefois connaître l'industrie touristique. On note que les agriculteurs de cette catégorie ont certaines difficultés ât établir une stratégie de commercialisation de leurs activités touristiques et à établir des liens avec des partenaires agricoles ou touristiques. Bien qu'ils parviennent à commercialiser leurs activités touristiques, ils intègrent difficilement les activités touristiques et agricoles à l'intérieur de leur système de gestion.

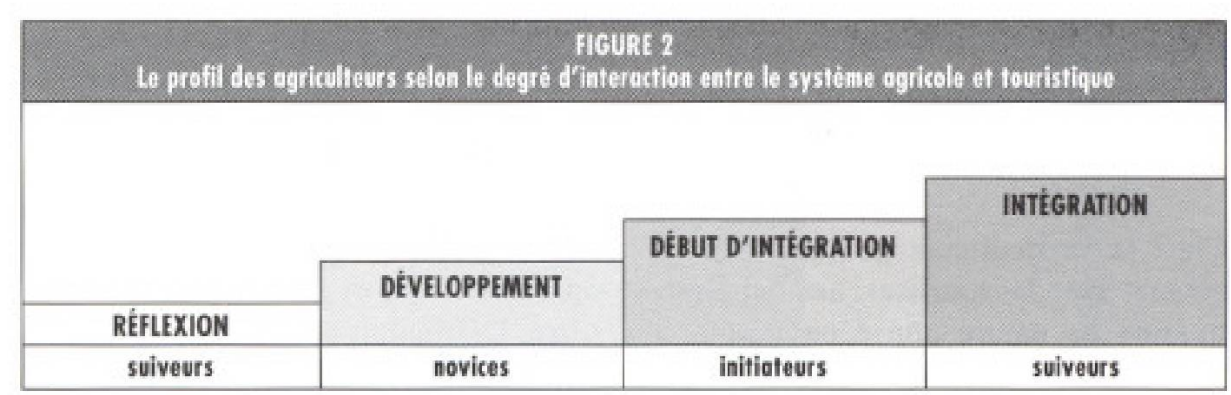

Les agriculteurs de la catégorie initiateurs du tourisme en agriculture offrent des activités touristiques basées principalement sur la vente des produits agricoles et lá visite des lieux de production de ces produits. Ce systène d'exploitation des activités touristiques n "est pas tellement organise, tant en ce qui concerne le contenu de l'offre touristique que sa commercialisation. Les clientèles visées sont principalement composées des résidants de la région ayant le potentiel d'acheter les produits agricoles de l'entreprise. Ces agriculteurs commencent à distinguer la clientèle des résidants et des touristes. Les agriculteurs de cette catégorie se sont tout d'abord tournés vers l'offre récréotouristique pour accroître les ventes de leurs produits agricoles sans nécessairement connâtre l'industrie touristique. Le manque de connaissance des initiateurs réduit grandement la commercialisation de leurs activités touristiques et l'établissement de liens avec des partenaires agricoles et touristiques. Ces agriculteurs ne parviennent alors pas vraiment à s'intégrer au touristiques.

Les agriculteurs de la catégorie suiveurs du tourisme en agriculture n'ont pas d'objectifs définis, de volonté d'aménagement précis de leur infrastructure, ni de démarche de diversification claire. Cette categorie est vulnérable financièrement. Dans pareil contexte, on cherche plutôt à maintenir l'entreprise agricole en vie par une diversification des activités, dont l'ajout d'activités touristiques. On doit noter l'absence d'efforts de promotion et de commercialisation des activités touristiques, ce qui n"est pas sans lien avec la précarité financière de ces entreprises. En consequence, ces activités ne semblent pas rentables et les agriculteurs de cette catégorie système de commercialisation des activités 
risquent de cesser leurs activités touristiques. Les agriculteurs de cette catégorie parviennent difficilement à integrer les activités touristiques et agricoles ă l'intérieur de leur système de gestion et ne font pas partie du systeme de commercialisation des produits touristiques. Ces entreprises sont celles qui sont les plus susceptibles d'abandonner leurs activites touristiques.

Cette étude exploratoire nous permet de poser les jalons du passage de l'entreprise agricole vers une entreprise agrotouristique. L'analyse du profil des agriculteurs qui se tournent vers le tourisme nous permet d'affirmer que les entreprises agricoles doivent passer d'une étape de réflexion à une étape de mise en action, puis de développement. En effet, le systême des professionnels, étape supérieure de la diversification, intègre la gestion des activités agricoles et des activités touristiques. A l'opposé, les agriculteurs de la catégorie suiveurs ont peu de chances de réussir parce que le système de gestion instauré n'établit aucun lien avec l'industrie touristique.

\section{Conclusion}

Face au phenomene de concentration et aux limites environnementales et de production qui y sont associées, les considerations 6conomiques apparaissent comme la principale motivation des agriculteurs a se tourner vers l'offre agrotouristique. En d'autres mots, les activites touristiques sont perçues comme une alternative au modèle productiviste qui exige des entreprises agricoles l'augmentation de la taille des exploitations. Ce type de diversification s'inscrit dans une perspective de développement durable où des agriculteurs refusent d'accroitre uniquement la production agricole, mais préfèrent se tourner vers d'autres activités permettant la croissance des revenus par la valeur ajoutée plutôt que par la croissance de la production. Cette recherche vient donc confirmer l"importance des considerations b́conomiques dans la diversification des activités agricoles.

Chez les agriculteurs qui sont dejà en contact avec les touristes, une autre motivation de nature non économique se manifeste. On trouve ainsi chez plusieurs de ces agriculteurs le desir de faire vivre aux touristes l'expérience a la ferme. Cette motivation est particulièrement présente chez les professionnels et se traduit par un désir de familiariser les touristes au monde agricole. L'agrotourisme devient alors une façon de présenter la réalité agricole et de véhiculer une image davantage positive des activités agricoles et de leurs retombées economiques.

Dans cette recherche, l'analyse des quatre catégories d'entreprises agricoles de la région Chaudière-Appalaches nous permet d'identifier des contraintes assoclés à l'offre agrotouristique. La première semble résider dans le manque de temps des agriculteurs pour développer, coordonner et promouvoir les activités touristiques en milieu agricole. Cette contrainte se retrouve même dans la catégorie de producteurs de loin la mieux développée et organisée, celle des professionnels du tourisme en milieu rural.

La deuxième contrainte est associée au manque de formation des agriculteur's. On observe en effet une méconnaissance de l' industrie touristique de la part des agriculteurs qui désirent commercialiser leurs activites touristiques. Les conséquences de cette méconnaissance sont nombreuses et comprenant, entre autres, une mauvaise commercialisation de l'offre agrotouristique et une absence de liens avec les intervenants de l'industrie touristique. Cette contrainte prend encore de l'importance quand on considère que les agriculteurs manquent de temps et devraient être plus efficients dans la gestion de leurs activités.

Finalement, une troisième contrainte apparaît associée au manque de soutien des agriculteurs qui developpent et gèrent leurs activités touristiques et ce, de façon plus ou moins isolée. Les associations ou les organisations agricoles et touristiques ne semblent pas offrit aux agriculteurs les outils nécessaires al cette forme de diversification. Les exploitants rencontrés sont d'ailleurs a la recherche de partenaires locaux pour compléter leur prestation agrotouristique et pour promouvoir leur offre. Ce soutien apparaît d'autant plus important si l'on considêre que la gestion et la nature des activités touristiques et agricoles peuvent différer. Bien que l'agriculteur doive considérer le caractêre saisonnier des activités touristiques et des activités agricoles, la planification et la commercialisation de chacune des activités ont des éléments spécifiques. De façon générale, lạ finalité de l'agriculture conduit vers la production d'un bien tangible, alors que la finalité de la production touristique conduit vers lá création d'un service. En consequence, l'agriculteur doit planifier la gestion de deux activités de nature différente. Cette planification exige non seulement une meilleure formation, mais un soutien de la part des réseaux agricoles et touristiques engagés dans ces activités.

Bien que cette recherche exploratoire ait été réalisée à partir d'une approche phênoménologique et qu'elle n'ait pas la prétention de dresser un portrait de la situation de l'ensemble des entreprises agricoles québecoises, elle permet de mieux comprendre le profil des agriculteurs en matiêre de tourisme. Si les activités touristiques apparaissent comme un outil de diversification complementaire aux activités agricoles, la réussite de cette diversification n'est pas à la portée des agriculteurs tant qu'ils n' adoptent pas le comportement des professionnels.

Laurent Bourdeau est professeur de marketing au Departement d'economie agroalimentaire et des sciences de la consommation de l'Universite Laval.

Maurice Doyon est professeur d'economie au même departemen.

Dorothé Donne est diplomee de l'Institut agroéconomique méditerranéen de Montpellier.

\section{Notes}

1 On peut expliquer le mécanisme de prix par la recherche de la baisse des coutts de production. Le premier agriculteur qui, par exemple, adopte une technologie réductrice de couts voit augmenter son revenu net par unité produite. Dans de telles conditions, cet agriculteur voudra assurément augmenter sa production. Réalisant les benéfices associés à cette technologie, d'autres agri- 
culteurs imiteront l'innovateur et augmenteront eux aussi leur niveau de production. A un certain point, le nombre d'agriculteurs ayant adopté la nouvelle technologie sera suffisant pour faire augmenter l'offre totale de façon significative. Si bien que, au prix initial, l'offre sera excédentaire à la demande. Le prix de la denrée agricole chutera donc pour reétablir un équilibre entre l'offre et la demande (Cochrane, 1974). Le résultat net sera que tous les agriculteurs recevront alors un prix moindre pour leur denréc. Ainsi, les consommateurs paient moins qu'avant et les agriculteurs qui n'ont pas encore adopté la nouvelle technologie voient leur revenu net diminuer. A long terme, les principaux gagnants sont donc les consommateurs et, a court terme, les premiers agriculteurs à avoir innové (Debertin, 1986).

2 Un prix réel ou un prix en dollar constant est un prix duquel l'inflation a été soustraite. Dans ce texte, l'indice des prix a la consommation (IPC) a été utilisé pour corriger les prix de l'effet de l'inflation.

3 Les contraintes de production se manifestent dans les secteurs où l'offre est réglementée. C'est donc dire que pour augmenter sa production un producteur doit acquérir un droit de produire sous forme de quota. Toutefois, le prix de ces quotas est souvent tellement élevé que toutc expansion devient particulièrement couteuse, voire non rentable. A titre d'exemple, le producteur laitier québécois qui achète du quota aujourd'hui peut $s^{\prime}$ attendre à travailler douze ans sans rémuneration de son travail et de son capital avant de voir les premiers effets positifs de son investissement (Levallois, 1998), Il faut ajouter que les contraintes environnementales sont appelés à prendre de plus en plus d'importance en agriculture (Gouin et Lagacé, 2000 ). Dejà, au Québec, la production porcine et de la production de volaille est souvent pointée du doigt comme étant une source importante de pollution diffuse des cours d'eau. Cela s'explique du fait que le modèle productiviste a eu comme conséquenoc de spécialiser à outrance la production, brisant le lien traditionnel entre le sol et les animaux. Si bien qu'une entreprise de volaille de taille moyenne au Québec peut ne posseder aucun sol. Or les dejections d'une telle entreprise sont équivalentes, en terme de phosphore, à celles d'une municipalité de 10000 personnes (Union Québécoise de la conservation de la nature, 2000).

4 Dans cette recherche, le terme touriste correspond aux individus qui se déplacent hors de leur résidence habituelle pour plus de vingt-quatre heures, résident à plus de cent kilomètres de leur lieu de résidence habituelle et passent au moins une nuit hor de cetté résidencè. L'utilisation de cette définition est conforme à la définition de Tinard (1992) et de Go et al. (1992). Les résidants représentent alors des individus qui effectueront une visite dans une ferme située à moins de cent kilomètres de leur lieu de résidence habituelle, qui se déplaceront pour se rendre à la ferme et qui ne dormiront pas hor's de leur licu de résidence habituelle.

5 Dans l'entrevue en profondeur, le chercheur interroge de façon plus ou moins structurée un sujet à la fois (Perrien et al. 1984). Pour les besoins de notre enquête, nous avons done interrogé individuellement vingt-huit agriculteurs.

6 Les fermes de l'echantillon appartiennent donc à région Chaudière-Appalaches où l'on trouve près de $18 \%$ de toutes les fermes du Quebec. Dans cette région fortement agricole, l'agriculture à temps partiel compte pour environ le tiers des entreprises agricoles. Celles-ci sont dites pluriactives, c'est-à-dire que dans ces entreprises l'agriculteur pratique au moins deux activités (Matthieu, 1995).

\section{Bibliographie}

Abdelahim, T. (1998), Methode d'analyse d'une zone rurale. Montpellier, Institut Agronomique Méditerranéen de Montpellier, 50 pages.

Churchill, G.A. (1991), Marketing Research : Methodological Fomdations, $5^{\text {e }}$ edition, Montreal, The Dryden Press, 1070 pages.

Cochrane, W. (1974), Farm Prices Myth and Reality, Greenwood Press, 189 pages.

Debertin, D. (1986), Agricultural Production Economics, Macmillan Publishing Company, 365 pages.

Disez, N. (1996), Agritourisme el développement territoriale: Exemple dans le massif central, Thèse de doctorat de l'Université Blaise Pascal de Clermont Ferrand II, Département de géographie, 241 pages.

Dion, S. (2001), Des activités complementaires prennen la vedette, Le cooperateur agricole. p. $32-42$.

Doyon, M., Y. Proulx, M. Morisset, D.-M. Gouin, et M. Frigon (2001), Ende sur les structures des entreprises agricoles et sur I'environnement économique et réglementaire québécois, Rapport de recherche, Département d'économie agroalimentaire et des sciences de la consommation, Université Laval

Fougerousse, C. (1996), Le renouveau rural: dependance or autonomie, Paris : L'Harmattan, 375 pages.

Gauthy M., et M. Senechal Vander (1998), Etude de marché, Deboek Université, 200 pages.

Go, F. M., D. Milne, et L.J.R. Whittles (1992), Communities as Destinations : A Marketing Taxonomy for the Effective Intplementation of Tourism Action Plan, 30 (4), p. 31-37.

Gouin, D.-M., el A. Lagacé (2000), Diagnos túque de l'industrie porcine québécoise, Rapport de recherche, Département d'économie agroalimentaire et des sciences de la consommation, Québec, Université Laval.

Groupe de recherche en économie et politique agricole (1999), Annuaire statistique porcin, Département d' économie agroalimentaire et des sciences de la consommation, Québec, Université Laval.

Groupement de Grenoble (1986), Les relations agriculture-tourisme, Institut public de recherche pour l'ingénierie et de l'environnement, Cémagref, $\mathrm{n}^{*} 207$.

Lussato, B. (1972), Modele cybernérique hommes entreprises, tome I : Imroduction critique aur theories d'organisation, Paris, Dunod, 192 pages.

Levallois, R. (1998), Faut-il encore miser sur l'achat de quota? Colloque de l'entrepreneur gestionnaire, Québec, Groupe en économie rural, Québec, Université Laval.

Matthieu, N. (1995), L'emploi rural, wne vitalité cachée, Paris, L'Harmattan, 183 pages.

Perrien, J., E.J. Chéron, et M. Zins (1984), Recherche en marketing : Methodes et décisions, Montréal, Gaẻtan Morin éditcur, 615 pages.

Sabourin, C. (1985), Le tourisme dans les régions rurales du Québec : bilan et perspectives, Thèse de doctorat en droit et économie du tourisme, Université de droit, d'économie et des sciences d'Aix-Marseille III, 601 pages. Tinard, Y. (1992), Le Tourisme, Economie of Management, Paris, Edition McGraw-Hill,

Union Québécoise de la conservation de la nature (2000), Sauve Garde: La crise environmementale de l'agriculure québécoise, Automne.

Union des producteurs agricoles (1999), Répertoire du monde rural et de ses activités, Tournée Champêtre, La terre de chez nous, 69 pages. 